\title{
The acronym superiority effect
}

\author{
SARAh Laszlo and Kara D. Federmeier \\ University of Illinois, Urbana-Champaign, Illinois
}

\begin{abstract}
The visual world is replete with noisy, continuous, perceptually variant linguistic information, which fluent readers rapidly translate from percept to meaning. What are the properties the language comprehension system uses as cues to initiate lexical/semantic access in response to some, but not all, orthographic strings? In the behavioral, electromagnetic, and neuropsychological literatures, orthographic regularity and familiarity have been identified as critical factors. Here, we present a study in the Reicher-Wheeler tradition that manipulates these two properties independently through the use of four stimulus categories: familiar and orthographically regular words, unfamiliar but regular pseudowords, unfamiliar illegal strings, and familiar but orthographically illegal acronyms. We find that, like letters in words and pseudowords, letters in acronyms enjoy an identification benefit relative to similarly illegal, but unfamiliar strings. This supports theories of visual word recognition in which familiarity, rather than orthographic regularity, plays a critical role in gating processing.
\end{abstract}

Visual word recognition occurs rapidly in skilled readers, and, despite its speed, is both accurate and robust to interference. A prominent view in the reading literature (see, e.g., McClelland \& Rumelhart, 1981) is that word recognition proceeds as quickly and accurately as it does only because it does not function in a simple feedforward manner. Instead, according to this view, phonological and lexical-semantic representations interact in a top-down fashion with orthographic input. Two important sources for such top-down constraint are familiarity (prior experience processing a given stimulus and/or one with similar properties) and regularity (the extent to which a given stimulus conforms to language-specific rules governing word formation).

Stimulus familiarity plays a central role in many connectionist models of visual word recognition (see, e.g., Harm \& Seidenberg, 2004). In such models, activation spreads continuously between orthographic input and preexisting phonological, lexical, and semantic representations, so that familiarity at every level contributes simultaneously to the recognition of a visually presented letter string. Regularity is not formally represented in most models of this type. Nevertheless, an advantage (or, in some cases, a cost) for regular items may be observed because they are often similar to many known words (i.e., they have large lexical neighborhoods). Such regularity effects arise through general mechanisms of similarity and are thus actually a subclass of familiarity effects.

In contrast, it is orthographic regularity that constitutes a key gating factor for word recognition in dual-route models (see, e.g., Coltheart, Rastle, Perry, Langdon, \& Ziegler, 2001). Two pathways are proposed: One is phonologically mediated, wherein explicit rules are used to translate orthography to phonology, from which lexical access can take place. The other is associative, in which orthography is directly linked to lexical entries. The neural and functional processes underlying the computations of the direct associative and phonological pathways in dualroute models are typically formulated as distinct (see, e.g., Jobard, Crivello, \& Tzourio-Mazoyer, 2003). Orthographic regularity critically determines which pathways are available to any given stimulus, because only regular items can be processed via phonological translation. Familiarity, instead, plays a role only during direct access; the rules used for phonological translation apply regardless of familiarity, thereby allowing for the processing of regular but unfamiliar stimuli, such as pronounceable pseudowords.

In a recent study, Laszlo and Federmeier (2007) used electrophysiology to examine the contributions of regularity and familiarity to the recognition of visually presented letter strings. We took advantage of the fact that the N400 component of the event-related potential (ERP) has been identified as a functionally specific marker of lexicosemantic access. Prior work has established that repetition effects on the N400 (i.e., facilitation, in the form of amplitude reduction for subsequent encounters with a given stimulus) can be elicited by regular stimuli, whether or not they are familiar (e.g., words, and pronounceable pseudowords, such as DAWK), but not by unfamiliar, illegal letter strings (e.g., GHTS). Given these results, it seemed that the processes of semantic access indexed by N400 repetition effects were best cued by orthographic regularity (see, e.g., Deacon, Dynowska, Ritter, \& Grose-Fifer, 2004). However, no study had examined effects for illegal items that were also familiar. Laszlo and Federmeier (2007) therefore added a stimulus class novel to this literature-familiar, but orthographically illegal, acronyms (e.g., DVD) - and found that these items did elicit $\mathrm{N} 400$ repetition effects that were indistinguishable in size, timing, and scalp distribu-

S. Laszlo, slaszlo2@uiuc.edu 
tion from those observed to words and pseudowords. These data demonstrate that computations of meaning from print can proceed in the absence of orthographic regularity, as long as the input is highly familiar.

However, Laszlo and Federmeier (2007) observed an intriguing dissociation between the pattern observed on the N400 repetition effects and that seen for the N400 response to the first presentation of items from the different stimulus classes. As shown in the left panel of Figure 1, words, pseudowords, and illegal acronyms all elicited statistically identical repetition effects on the N400, whereas there were no repetition effects for unfamiliar, illegal strings. However, as shown in the right panel of Figure 1, there was no discriminable difference in the morphology of the waveforms elicited by familiar acronyms and illegal strings of letters on first presentation alone.

The first-presentation waveforms elicited by illegal strings and familiar acronyms may have been similar to one another (and different from those elicited by words and pseudowords) because the illegal stimulus classes are considerably lower in lexical density, a factor known to affect N400 amplitude (Holcomb, Grainger, \& O'Rourke, 2002). In the present study, we sought to more completely test the hypothesis that familiar acronyms undergo lexical access processes similar to those engaged by regular items such as words and pseudowords. To achieve this, we again fully crossed the factors of familiarity and regularity in a paradigm more suited to exploring the linguistic processes engaged by a single presentation of a visual stimulus.

The Reicher-Wheeler task has been a reliable tool for demonstrating top-down influences on visual word recognition for more than 35 years (since Reicher, 1969). The well-established result is that letters in briefly presented words are identified more accurately than letters in briefly presented nonwords or letters presented alone. This is true even when guessing strategies are ruled out by providing two viable alternatives for a forced-choice response (e.g., querying " $\mathrm{D}$ " or " $\mathrm{K}$ " in the last position of the target word WORK). This word superiority effect has classically been interpreted as evidence against strictly feedforward accounts of word recognition, and it has been successfully modeled with interactive networks (e.g., McClelland \& Rumelhart, 1981), wherein the identification benefit for letters in words indeed accrues from the top-down spread of activation from high-level representations (which are not present for illegal strings of letters). Thus, word-superiority-effect results and models both suggest that preexisting representations facilitate the processing of familiar letter strings, even in the relatively low-level task of letter identification.

What is not clear from either data or simulations is the precise nature of the high-level representations involved. One possibility is that they are really just lexical entriesthat is, that words are processed more accurately by virtue of inclusion in a literal list of familiar strings marked for expedited processing. A second possibility is that the orthographic regularity of words and pseudowords (for which superiority effects are also observed; Baron \& Thurston, 1973; Carr, Davidson, \& Hawkins, 1978) provides access to phonological representations that are the basis of the top-down effects. This second possibility is likely preferred by adherents of dual-route accounts of visual word recognition, because it is otherwise difficult for a noninteractive model to explain superiority effects for items such as pseudowords, which do not have lexical entries.

To date, no study in the Reicher-Wheeler tradition has evaluated the independent contributions of orthographic regularity and stimulus familiarity to visual word recognition. This reflects the usual stimulus sets, which include words (regular and highly familiar), pseudowords (regular and unfamiliar on a whole-item level), and illegal letter
A

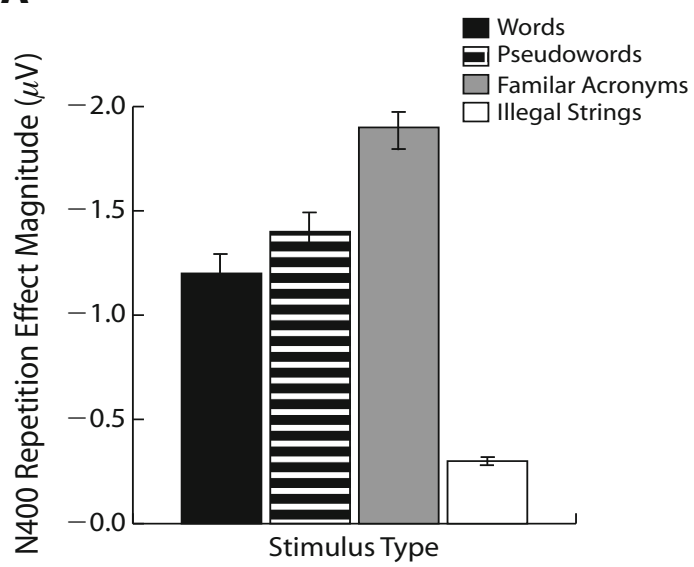

B

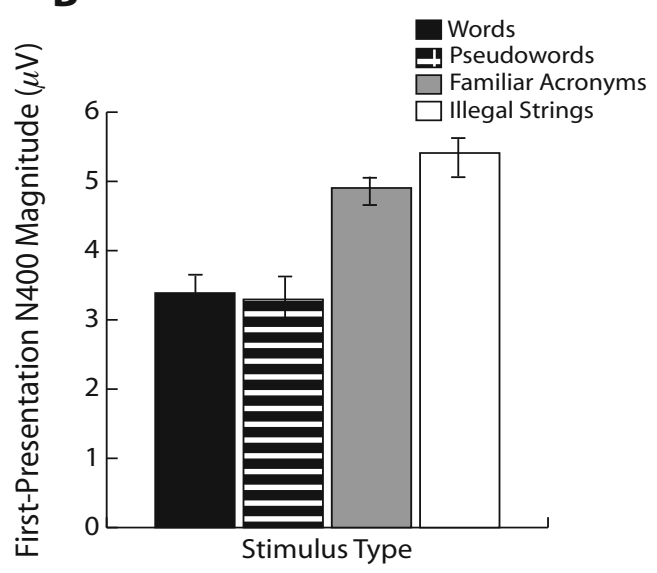

Figure 1. Data from Laszlo and Federmeier (2007). As can be seen in panel A, N400 repetition effect size for words, pseudowords, and familiar acronyms is similar (does not differ statistically, though the effect for acronyms is numerically larger than that for words or pseudowords), whereas ERP amplitude in the N400 window does not change with repetition of unfamiliar illegal strings. Repetition effect sizes are plotted negative up, on the $y$-axis. As can be seen in panel $B$, single-presentation $N 400$ amplitude for words and pseudowords, which are regular, differs from single-presentation N400 amplitude for familiar acronyms and illegal strings, which are not. Error bars represent the standard error of the means. 
strings (neither regular nor familiar). In the present experiment, we employed fully crossed stimuli, such as those employed by Laszlo and Federmeier (2007), in a ReicherWheeler paradigm, now including acronyms as a critical familiar, but illegal, class. By doing so, we could assess the independent contributions of regularity and familiarity to superiority effects in visual word recognition, and we could also address concerns about the similarity of the firstpresentation waveforms elicited by illegal strings and familiar acronyms in the Laszlo and Federmeier ERP study.

Several previous studies (Henderson, 1974; Noice \& Hock, 1987; Prinzmetal \& Millis-Wright, 1984) have used acronyms as stimuli with rapid serial visual presentation. Although these studies were each concerned with slightly different cognitive questions, they all broadly suggested that familiar acronyms are processed at what might be called a lexical level. However, all these studies differed from the canonical Reicher-Wheeler paradigm in important ways, which makes their interpretation difficult, at least with respect to the current question about the representations that subserve visual word recognition.

In this experiment, therefore, we presented words, pseudowords, familiar illegal acronyms, and illegal (nonacronym) letter strings in a traditional Reicher-Wheeler paradigm. If superiority effects primarily reflect orthographic regularity, then we should replicate past studies, observing superiority effects for words and pseudowords, but no benefit for letter recognition in acronyms. However, if familiarity is the more important component to semantic access, as the Laszlo and Federmeier (2007) ERP data suggest, we should observe superiority effects in the Reicher-Wheeler task for familiar acronyms, relative to similarly illegal but unfamiliar letter strings.

\section{METHOD}

\section{Participants}

Data were analyzed from 61 participants ( 19 female, mean age 19 years, 25 in the primary experiment and 18 in each of two control experiments) recruited from the University of Illinois; 9 additional participants were excluded for having insufficient acronym knowledge (described below). All were neurologically normal, right-handed, monolingual speakers of English, with normal or corrected-to-normal vision.

\section{Stimuli}

Critical stimuli were three- to four-letter strings from four categories (22 items in each): acronyms, illegal strings, words (high and low frequency), and pseudowords. Filler acronyms and illegal strings (22 of each) were also presented, but data from those items were not analyzed. Acronyms were composed of all consonants or all vowels, and were considered familiar to our participant pool on the basis of a norming study conducted with 236 undergraduates at the University of Illinois and individual questionnaires assessing acronym knowledge administered to each participant after behavioral data collection. Nine participants who were unable to correctly identify at least $70 \%$ of critical acronyms were excluded from analysis. Because multiple written-frequency corpora were used (Dennis, 1995; Kučera \& Francis, 1967) in order to obtain a viable frequency measure for acronyms, words, pseudowords, and illegal strings, we report here written probability, defined as the written frequency of a stimulus divided by the number of items surveyed in the corpus from which that stimulus was selected. Acronym mean written probability was .00000071 (minimum 0, maximum .0000039). Due to the extremely limited number of possible familiar one-off acronym pairs, each participant saw both components of each acronym pair (e.g., HDTV and HGTV).

Illegal strings were composed of all consonants and did not represent any acronym familiar to our participant pool. Although written frequency was not available for illegal strings, wildcard analysis (i.e., for the stimulus GHTS, summing the frequency of every occurrence of the string GHTS in any word in the frequency corpus) indicated a mean written probability of .0015 (minimum 0 , maximum $.01)$. Similar wildcard analysis conducted on our acronyms indicated a mean written probability of .0001 (minimum 0, maximum .001).

Words had a mean written probability of .00004 (minimum .0000004, maximum .0003).

Pseudowords were created by choosing one low-frequency word not used as an experimental item and replacing the first or last letter in order to produce a pronounceable nonword. Wildcard analysis indicated that none of these items appeared in the corpora surveyed.

In two additional control experiments, filler illegal items were replaced with categories of illegal strings rigorously matched to our acronyms for perceptual characteristics: items (Experiment 2) that were response and (partially) flanker matched (e.g., NHL, matched, across participants, with NHJ and MHL; "H" and "F" queried in Position 2, in each case) and, to mitigate against the repetition engendered by that control, items (Experiment 3 ) that were response matched only (e.g., LSD matched with PSV; "S" and "C" queried in Position 2 in each; flankers chosen randomly without replacement from the flankers of the targets in the acronym category). These categories of illegal strings, however, were lower in written probability than were the original illegals (.00000045 and .000034 , respectively).

\section{Procedure}

Each participant was presented with each stimulus exactly once in a randomly ordered list (individually randomized for each participant), with three evenly spaced rest breaks. Before the main experimental session, the participants were given a demonstration of trial structure, and engaged in a practice block with stimuli similar to those in the experimental lists. The practice block also implemented a psychophysical stair-stepping procedure by which targetpresentation duration was individualized for each participant so that participants were performing as near as possible to $70 \%$ overall letter identification accuracy (range, $56-87 \mathrm{msec}$; mean, $69 \mathrm{msec}$ ). During the practice, but not during the main experimental session, participants were notified with beeps when they made errors.

An ampersand pattern mask preceded and followed each stimulus, and a warning cross preceded the forward pattern mask. The backward pattern mask was followed by the response cue, which consisted of one ampersand for each letter in the previously presented stimulus, one of which was highlighted in a red box. The two alternatives for the Reicher task were presented above and below the red box. Participants indicated with a buttonpress which alternative had occurred in the highlighted position in the previously presented item. Importantly, for all classes of stimuli, both alternatives presented in the response cue were viable completions in the same category as the stimulus that was actually presented. Illustrative examples are displayed in Table 1. The position of the correct and incorrect probe letter was randomized on each trial for each participant. Across the stimulus set (but not within items), all four possible letter positions were queried. The response cue remained on the screen until a response was made, and then the next trial began immediately.

\section{RESULTS}

Performance on the control categories of strings (from Experiments 2 and 3 ) did not differ from performance on the illegal strings in Experiment 1, as indicated by planned $t$ tests [for Experiment 2, $t(17)=.75, p=.23$; for Experiment $3, t(17)<.0001, p>.999]$. Given this, and the fact that the composition of illegal strings was the only difference across Experiments 1-3, the analyses that follow 


\begin{tabular}{|c|c|c|c|c|}
\hline \multicolumn{5}{|c|}{$\begin{array}{c}\text { Table 1 } \\
\text { Illustrative Examples of Target Stimuli, } \\
\text { Probe Displays, and Forced-Choice Completions } \\
\text { for All Four Critical Stimulus Categories }\end{array}$} \\
\hline Stimulus Type & Target & Probe & Alternative 1 & Alternative 2 \\
\hline Word & DUCT & $\begin{array}{r}\mathrm{K} \\
\& \& \& \&\end{array}$ & $\mathrm{~T}$ (DUCT) & K (DUCK) \\
\hline Pseudoword & DAWK & $\begin{array}{r}\mathrm{T} \\
\mathrm{W} \\
\& \& \& \& \\
\mathrm{D}\end{array}$ & D (DAWK) & W (WAWK) \\
\hline Acronym & HDTV & \&\&\&\& & D (HDTV) & G (HGTV) \\
\hline Illegal String & GHTS & $\begin{array}{r}\mathrm{D} \\
\mathrm{C} \\
\& \& \& \& \\
\mathrm{H}\end{array}$ & H (GHTS) & $\mathrm{C}$ (GCTS) \\
\hline
\end{tabular}

Note-As is evident in the Alternative 1 and Alternative 2 columns, no matter which of the two probe letters is selected, an item from the same stimulus class as the target is completed.

collapse across experiment unless otherwise noted. Mean letter identification accuracies were: words, .88 $(\sigma=.07)$, pseudowords, $.84(\sigma=.1)$, acronyms, $.77(\sigma=.10)$, and illegal strings, $.73(\sigma=.11)$. These results are summarized in Figure 2, which also presents a breakdown of letter identification performance by illegal string category.

One-way repeated measures ANOVAs were conducted over both participants and items, with five levels of stimulus type: high-frequency words, low-frequency words, pseudowords, acronyms, and illegal strings. The illegal strings level of the item-based ANOVA was limited to data from the original (high-frequency) strings, because only these were presented to participants in all three experiments. Both ANOVAs revealed significant effects of stimulus type [for participants, $F(4,240)=47.39, p<.0001$; for items, $F(4,84)=12.67, p<.0001]$. For subsequent tests, words were collapsed over frequency. Follow-up $t$ tests indicated that mean accuracy was higher for words than for any other stimulus category [words vs. pseudowords, $t(60)=2.5, p=.02$; words vs. acronyms, $t(60)=$ $7.5, p<.0001$; words vs. illegal strings, $t(60)=10.5, p<$ $.0001]$. Accuracy was also higher for pseudowords than for acronyms $[t(60)=4.2, p<.0001]$.

To evaluate superiority effects, letter identification accuracy for words, pseudowords, and acronyms was compared with that for illegal strings. Letters in each of those categories of stimuli were identified more accurately than were letters in illegal strings, as indicated by follow-up $t$ tests [for words, $t(60)=10.5, p<.0001$; for pseudowords, $t(60)=6.76, p<.0001$; for acronyms, $t(60)=2.4$, $p=.02]$. Effect size was quantified with a standardized difference between means, which we denote here as $d^{\prime}$. The superiority effect was largest for words $\left(d^{\prime}=1.20\right)$, then pseudowords $\left(d^{\prime}=.82\right)$, and then acronyms $\left(d^{\prime}=.30\right.$.)

\section{DISCUSSION}

In this study, we tested for word superiority effects using (1) familiar, regular words, (2) unfamiliar, regular pseudowords, (3) familiar, illegal acronyms, and (4) unfamiliar illegal strings. We predicted that, in the absence of regularity, stimulus familiarity could initiate contact with high-level representations in the language comprehension network. If so, we would observe superiority effects in letter identification for familiar acronyms, similar to those observed for words and pseudowords. Such a result would support accounts of the word superiority effect that emphasize familiarity over rule-governedness.

We replicated previous results, finding that briefly presented letters in words and pronounceable pseudowords were identified more accurately than were letters in control strings, even when both forced-choice alternatives created viable instances of the target class (e.g. " $D$ " or " $K$ " for the target word WORK). Critically, we also observed a superiority effect for illegal, but familiar acronyms. Briefly presented letters in acronyms were better recognized, relative to letters in similarly illegal, but unfamiliar, strings. As with the words, this superiority effect occurred even though both response alternatives created familiar acronyms (e.g., "D" or "G" for the target HGTV).

Our results complement those of Noice and Hock (1987), who observed an identification benefit for illegal acronyms in a modified Reicher-Wheeler paradigm wherein participants answered "yes" or "no" to probe letters following targets. Because incorrect probe letters generally did not create viable acronyms in that study, participants might have occasionally arrived at correct answers by implementing a guessing strategy. In the present study, such a systematic guessing strategy was not possible. Our observation of an acronym superiority effect thus provides strong evidence that top-down benefits, as commonly observed for words and pseudowords, also occur for orthographically illegal items, if those items are familiar.

Rumelhart and McClelland (1982) also observed a superiority effect for irregular items with high lexical densities (e.g., SLRT). In their model, such items receive facilitation via their similarity to lexically represented words; a similar account based on regularity could also be advanced, since subparts of these items map onto frequent phonological combinations that could be parsed by a rule-based translation system. However, the acronyms in our study had a mean lexical density of .86 (with 0-3 lexical neighbors), making it difficult to explain their recognition benefits as reflecting similarity to known words or frequent phonological combinations. Indeed, the acronyms were just as irregular as the control strings, and did not differ from them in mean lexical density. Nevertheless, they were processed more accurately in the Reicher-Wheeler task. We therefore conclude that top-down benefits to recognition can accrue if the orthographic input is similar to at least one item represented in the lexicon, and that the lexicon contains illegal strings if these are familiar and linked to meaning.

Our observation of an acronym superiority effect for items given a single presentation is also in agreement with the claim by Laszlo and Federmeier (2007) that the acronym repetition effects on the $\mathrm{N} 400$ reflected facilitated lexico-semantic access. In that study, the first-presentation morphology of the ERP elicited by illegal acronyms was remarkably similar to that elicited by unfamiliar illegal strings, a fact that may have been due to the low lexical density of the familiar acronyms. The present result makes 


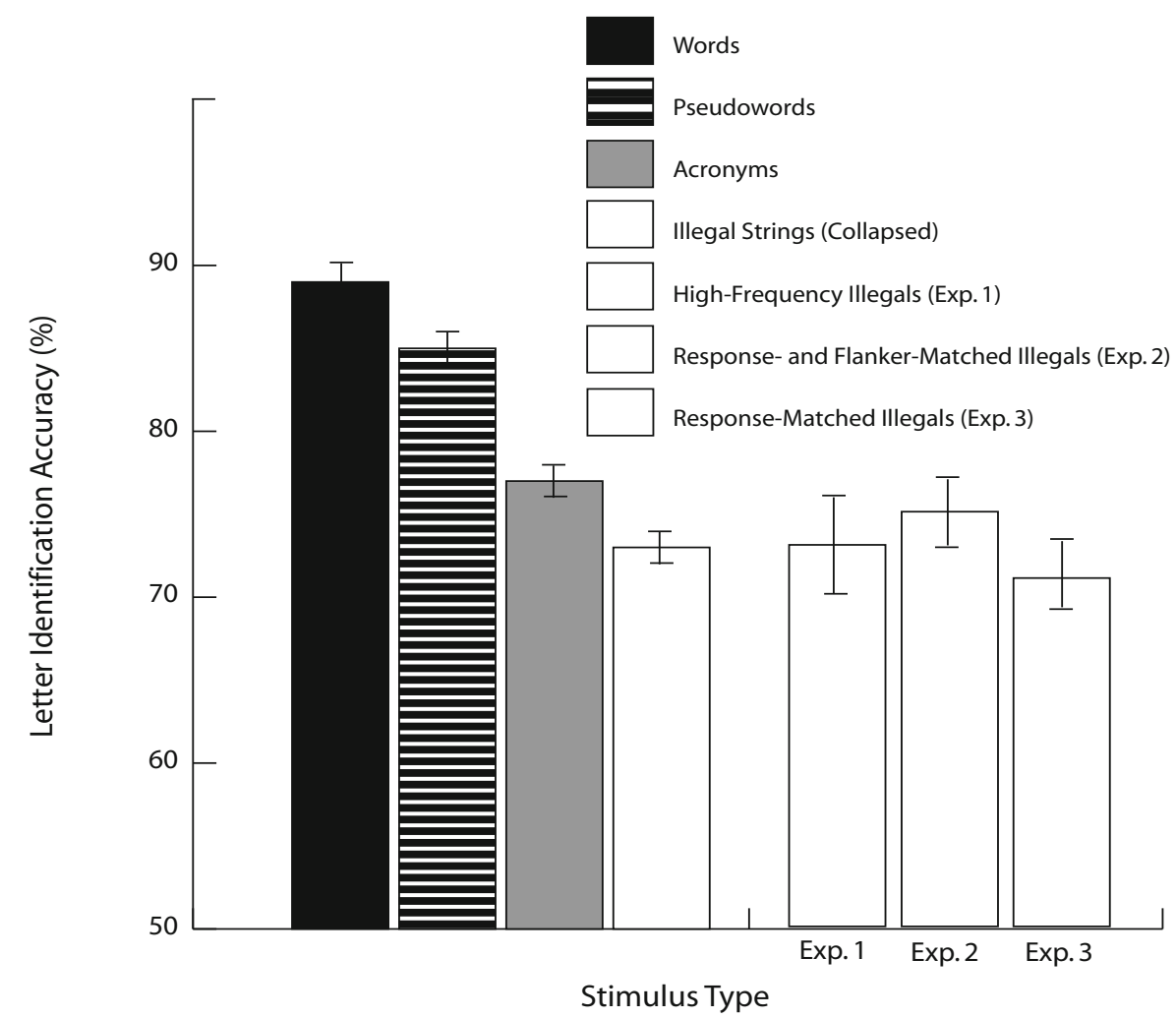

Figure 2. Mean percentage letter identification accuracy collapsed across Experiments 1-3 for words, pseudowords, acronyms, and illegal strings, as well as breakdown of illegal string performance by experiment. Performance is statistically best for words, then pseudowords, and then acronyms, with performance being worst for illegal strings. Performance is identical on all three categories of illegal strings. Error bars represent the standard error of the means.

clear that familiarity can facilitate the processing of illegal stimuli, even on a single presentation.

Another striking aspect of the present results is that the superiority effect was larger for words, relative to both pseudowords and acronyms. This result may have arisen because our words were more frequent than these other stimuli. However, past studies have not observed frequency effects on Reicher-Wheeler task performance (Günther, Gfroerer, \& Weis, 1984). Furthermore, in the present study, control strings actually had a higher mean written probability than the acronyms, but were still recognized less accurately. Another intriguing possibility is that acronyms receive direct benefits from their lexical entries, whereas pseudowords receive indirect benefits via their resemblance to lexical neighbors. Words would receive top-down support from both their lexical entries and their neighbors. This possibility is in line with interactive models of visual word recognition (see, e.g., Harm \& Seidenberg, 2004), wherein orthographic, phonological, and semantic representations of stimuli participate interactively in recognition. Interactive models allow for additive benefits of familiarity (i.e., having preexisting lexical representations) and similarity (which, in these models, subsumes regularity). However, in competing models, wherein recognition proceeds as a horse race between processes sensitive to regularity and processes sensitive to familiarity, the present results are not easily explained.

\section{AUTHOR NOTE}

This research was supported by NIMH Training Grant T32 MHI9554 to S.L. and NIA Grant AG26308 to K.D.F. The authors acknowledge G. Dell and E. Wlotko for helpful discussion, and C. Sung for data collection. Correspondence concerning this article should be addressed to S. Laszlo, Beckman Institute, University of Illinois, 405 N. Mathews Avenue, Urbana, IL 61801 (e-mail: slaszlo2@uiuc.edu).

\section{REFERENCES}

Baron, J., \& Thurston, I. (1973). An analysis of the word-superiority effect. Cognitive Psychology, 4, 207-228.

Carr, T. H., Davidson, B. J., \& Hawkins, H. J. (1978). Perceptual flexibility in word recognition: Strategies affect orthographic computation but not lexical access. Journal of Experimental Psychology: Human Perception \& Performance, 4, 674-690.

Coltheart, M., Rastle, K., Perry, C., Langdon, R., \& Ziegler, J. (2001). DRC: A dual route cascaded model of visual word recognition and reading aloud. Psychological Review, 108, 204-256.

Deacon, D., Dynowska, A., Ritter, W., \& Grose-Fifer, J. (2004). Repetition and semantic priming of nonwords: Implications for theories of N400 and word recognition. Psychophysiology, 41, 60-74.

Dennis, S. (1995). The Sydney Morning Herald word database. Retrieved October 2004 from www2.psy.uq.edu.au/CogPsych/Noetica/ OpenForumIssue4/SMH.html.

Günther, H., Gfroerer, S., \& Weis, L. (1984). Inflection, fre- 
quency, and the word superiority effect. Psychological Research, 46, 261-281.

Harm, M. W., \& Seidenberg, M. S. (2004). Computing the meanings of words in reading: Cooperative division of labor between visual and phonological processes. Psychological Review, 111, 662-720.

HENDERSON, L. (1974). A word superiority effect without orthographic assistance. Quarterly Journal of Experimental Psychology, 26, 301-311.

Holcomb, P. J., Grainger, J., \& O'Rourke, T. (2002). An electrophysiological study of the effects of orthographic neighborhood size on printed word perception. Journal of Cognitive Neuroscience, 14, 938-950.

Jobard, G., Crivello, F., \& Tzourio-Mazoyer, N. (2003). Evaluation of the dual route theory of reading: A metanalysis of 35 neuroimaging studies. NeuroImage, 20, 693-712.

KuČERA, H., \& FranCIS, W. N. (1967). Computational analysis of presentday American English. Providence, RI: Brown University Press.

Laszlo, S., \& Federmeier, K. D. (2007). Better the DVL you know:
Acronyms reveal the contribution of familiarity to single word reading. Psychological Science, 18, 122-126.

McClelland, J. L., \& Rumelhart, D. E. (1981). An interactive activation model of context effects in letter perception: Part I. An account of basic findings. Psychological Review, 88, 375-407.

NoICE, H., \& Hock, H. S. (1987). A word superiority effect with nonorthographic acronyms: Testing for unitized visual codes. Perception \& Psychophysics, 42, 485-490.

Prinzmetal, W., \& Millis-Wright, M. (1984). Cognitive and linguistic factors affect visual feature integration. Cognitive Psychology, 16, 305-340.

Reicher, G. M. (1969). Perceptual recognition as a function of meaningfulness of stimulus material. Journal of Experimental Psychology, 81, 274-280.

Rumelhart, D. E., \& McClelland, J. L. (1982). An interactive activation model of context effects in letter perception: Part 2. The contextual enhancement effect and some tests and extensions of the model. Psychological Review, 89, 60-94.

\section{APPENDIX}

\begin{tabular}{lccc}
\hline Acronym & $\begin{array}{c}\text { Written } \\
\text { Probability }\end{array}$ & $\begin{array}{c}\text { Lexical } \\
\text { Density }\end{array}$ & $\begin{array}{c}\text { Acronym Familiarity } \\
\text { Proportion of Correct } \\
\text { Responses, } N=61 \text { ) }\end{array}$ \\
\hline HGTV & 0 & 0 & .79 \\
HDTV & .00000017 & 0 & .92 \\
BBC & .00000013 & 0 & .79 \\
NBC & .00000260 & 0 & .99 \\
NHL & .00000017 & 1 & .99 \\
NFL & .00000270 & 1 & .00 \\
LSD & .00000130 & 2 & .94 \\
LCD & .00000110 & 2 & .71 \\
MSG & .00000013 & 1 & .73 \\
MPG & .00000013 & 1 & .94 \\
JPC & .00000013 & 0 & .76 \\
PVC & .00000013 & 0 & .72 \\
CBS & .00000038 & 0 & .99 \\
PBS & .00000390 & 0 & .99 \\
PMS & .00000140 & 1 & .99 \\
STD & .00000038 & 1.00 \\
LTD & .00000013 & 3 & .40 \\
THC & .00000013 & 2 & .66 \\
TLC & .00000013 & 3 & .91 \\
WWI & .00000013 & 1 & .79 \\
WWW & .00000013 & 0 & .94 \\
\hline
\end{tabular}

(Manuscript received August 4, 2006;

revision accepted for publication February 20, 2007.) 